\title{
Neuste Erkenntnisse
}

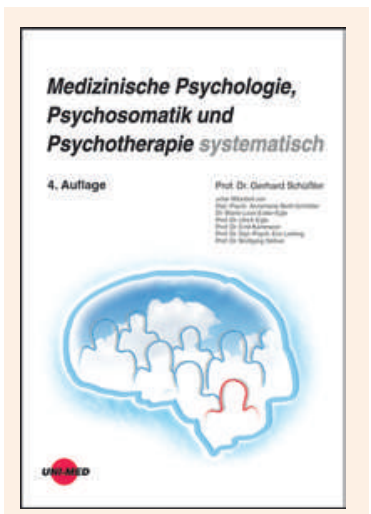

Gerhard Schüßler (Hrsg.) Medizinische Psychologie, Psychosomatik und Psychotherapie systematisch

Bremen, London, Boston: Uni-Med; 2011

288 Seiten, 4., neubearb. Auflage, 66 Abb. 32.80 Euro ISBN 978-3-8374-1227-7
Dieses Lehr- und Lernbuch, das in einer erweiterten und vollständig überarbeiteten 4. Auflage erschienen ist, bietet einen integrativen und umfassenden Einblick in die Grundlagen und neuesten Ergebnisse der Medizinischen Psychologie, Psychosomatischen Medizin und Psychotherapie. Auf nur 288 Seiten wird auf die Klinik, die psychosomatischen Faktoren, therapeutischen Massnahmen und die Psychotherapie der wichtigsten Krankheitsbilder eingegangen: Depression, Angst, Sexualstörungen, Schlafstörungen, Somatisierung, Psychoonkologie, Essstörungen, Schmerz und die psychosomatischen Störungsbilder der jeweiligen medizinischen Fachgebiete (Innere Medizin, Gynäkologie/Geburtshilfe, HNO, Dermatologie, Neurologie, Augenheilkunde, Urologie, Pädiatrie und Zahnheilkunde).

Ausgehend von den Grundlagen der ärztlichen Gesprächsführung, werden die relevanten psychotherapeutischen Richtungen Tiefenpsychologie (Psychodynamische Psychotherapie), Verhaltenstherapie, Entspannungsverfahren, Paar-/Familientherapie und Kinder-/Jugendpsychotherapie knapp und anschaulich erläutert.

Das didaktische Konzept der Lehrbuchreihe des Uni-Med Verlags sorgt mit seinem Schwarz-RotZweifarbendruck für eine klare Systematik in komprimierter Darstellung mit vielen Abbildungen, Schemata und Tabellen, was diesem Lernbuch eine gute Übersichtlichkeit verleiht. Als Nachschlagewerk ist es vielleicht weniger geeignet, da das Register zu beschränkt ausgefallen ist - ein Kompromiss, den man aber in der Zeit des Internets in Kauf nimmt. Der Aufbau ist gutstrukturiert, sodass sich der Leser mit Hilfe des Inhaltsverzeichnisses im Buch schnell zurechtfindet.

Die kondensierten und knapp gehaltenen Formulierungen führen manchmal dazu, dass der Text nicht immer flüssig lesbar ist und gewisse Passagen mehrfach gelesen werden müssen. Insofern erinnert dieses Buch oftmals auch an die komprimierten Skripten, wie wir sie für die Staatsvorbereitungen einsetzten. Andererseits imponiert dieses Buch, weil wirklich alle neusten Erkenntnisse berücksichtigt sind, die Krankheitsbilder und die allgemeinen therapeutischen Massnahmen umfassend beschrieben werden. Dabei bezieht sich der Autor auf die neusten Standard- und Übersichtswerke von Uexküll und Senf/Broda aus dem Jahr 2011 und fügt eine ausgewählte Literaturliste und eine etwas rudimentär ausgefallene Liste der wichtigsten Internet-Adressen hinzu.

Ich kann das Buch allen psychosomatisch arbeitenden Kollegen als Manual wärmstens empfehlen und ebenso all jenen, die sich mit schwierigen Patienten schwertun und an Psychosomatik interessiert sind, aber keinen «dicken Schunken wälzen» wollen.

Dr. med. Pierre Loeb, Basel 